\title{
SKEW POLYNOMIALS AND ALGEBRAIC REFLEXIVITY
}

\author{
NICOLE SNASHALL and J. F. WATTERS \\ Department of Mathematics and Computer Science, University of Leicester, Leicester LE1 7RH, England
}

(Received 16 January, 2001; accepted 7 May, 2002)

\begin{abstract}
For an arbitrary $K$-algebra $R$, an $R, K$-bimodule $M$ is algebraically reflexive if the only $K$-endomorphisms of $M$ leaving invariant every $R$-submodule of $M$ are the scalar multiplications by elements of $R$. Hadwin has shown for an infinite field $K$ and $R=K[x]$ that $R$ is reflexive as an $R, K$-bimodule. This paper provides a generalisation by giving a skew polynomial version of his result.
\end{abstract}

2000 Mathematics Subject Classification. Primary 16D20, 16S36.

1. Introduction. If $V$ is a vector space over a field $K$ and $\mathcal{L}$ is a lattice of subspaces then $\operatorname{alg} \mathcal{L}$ is defined to be the algebra of all $K$-endomorphisms of $V$ which leave $\mathcal{L}$ point-wise fixed; dually if $R$ is a subalgebra of End ${ }_{K} V$, then lat $R$ is defined to be the lattice of all subspaces of $V$ which are left invariant by every element of $R$. Combining these two functors produces alg (lat $R$ ), an algebra containing $R$, and $R$ is called reflexive when these two algebras are equal. Thus, an algebra of operators on a vector space is called reflexive when no larger algebra of operators has the same lattice of invariant subspaces. This terminology originated with Halmos [3] although earlier work also explored some of this area; for example, the lattice of invariant subspaces of a single linear transformation was studied in [1].

In [2] a start was made on studying an extension of the concept of alg lat where the focus moved to classes of representations, that is $R, K$-bimodules, of a $K$-algebra $R$. For an arbitrary $K$-algebra $R$ a bimodule ${ }_{R} M_{K}$ is said to be (algebraically) reflexive if, whenever $\alpha \in$ End $_{K} M$ is such that $\alpha m \in R m$ for all $m \in M$, then there is an $r \in R$ such that $\alpha$ is simply left multiplication by $r$.

More generally, define for rings $R, A$ and bimodule ${ }_{R} \mathrm{M}_{A}$ [2]

$$
\text { alglat } M:=\left\{\alpha \in \text { End } M_{\mathrm{A}} \mid \alpha m \in R m \text { for all } m \in M\right\},
$$

and let $\lambda(r) \in \operatorname{End}_{K} M$ send $m$ to $r m$ for all $m \in M$, setting

$$
\lambda(R):=\{\lambda(r): r \in R\},
$$

then $\lambda(R) \subseteq$ alglat $M$ and $M$ is reflexive when this is equality. In Halmos' notation alglat $M$ becomes alg (lat $\lambda(R))$ and ${ }_{R} M_{K}$ is a reflexive bimodule exactly when $\lambda(R)$ is a reflexive algebra of operators on ${ }_{R} M_{K}$. It is of interest to note that Leptin [5] considered the concept of alglat, although not in this notation, for topological modules in a study of completeness and linear compactness.

A central lemma in the paper by Hadwin [4] states that if $K$ is an infinite field and $R=K[x]$, the polynomial ring, then the algebra ${ }_{R} R_{K}$ is reflexive as an $R, K$-bimodule. It is the aim of this paper to establish a skew polynomial version of this result. 


\section{Results.}

LEMMA 1. Let $M=R m$ be an $R$, A-bimodule with A a subring of $R$ such that $k m=m k$ for all $k \in A$. Suppose there is a set $\mathcal{E}$ of additive group homomorphisms from $R m$ into Am such that

(1) $\epsilon(r v)=\epsilon(r \epsilon(v))$ for all $r \in R, v \in M$;

(2) $\epsilon(u)=0$ for all $\epsilon \in \mathcal{E}$ implies $u=0$.

Then ${ }_{R} M_{A}$ is reflexive.

Proof. Let $\alpha \in$ alglat $M$. Then $\alpha m=a m$ for some $a \in R$, and $\alpha-\lambda(a)=\beta \in$ alglat $M$ with $\beta m=0$. We show that $\beta=0$, whence $\alpha=\lambda(a)$.

Let $u \in M$. Then $\beta u=r u$ for some $r \in R$. Also, for all $\epsilon \in \mathcal{E}$, there is $s \in R$ with $\beta(u-\epsilon(u))=s(u-\epsilon(u))$. Hence, $r u-\beta(\epsilon(u))=s u-s \epsilon(u)$. Now $u \in M$ so $\epsilon(u)=k m$ for some $k \in A$, so $\beta(\epsilon(u))=\beta(k m)=\beta(m k)=\beta(m) k=0$. Thus $r u=s u-s \epsilon(u)$. Therefore $\epsilon(r u)=\epsilon(s u-s \epsilon(u))=\epsilon(s u)-\epsilon(s \epsilon(u))=\epsilon(s u)-\epsilon(s u)$ for all $\epsilon \in \mathcal{E}$. By (2) $r u=0$, so $\beta u=0$ for all $u \in U$ and so $\beta=0$.

THEOREM 1. Let $R=A[x ; \sigma, \delta]$ be a skew polynomial ring over a domain $A$ with $\sigma: A \rightarrow A$ a monomorphism and $\delta$ a $\sigma$-derivation on $A$. Let $K=\{a \in Z(A) \mid \sigma a=a$, $\delta a=0\}$. If $K$ is infinite, then ${ }_{R} R_{A}$ is reflexive.

Proof. We apply Lemma 1 to the $R, A$-bimodule $R$, so $m=1$. For each $k \in K$ define the evaluation map $\epsilon_{k}: R \rightarrow A$ by

$$
\epsilon_{k}: \sum_{i=0}^{n} r_{i} x^{i} \mapsto \sum_{i=0}^{n} r_{i} k^{i}, \quad r_{i} \in A .
$$

It is clear that $\epsilon_{k}$ is an additive group homomorphism from $R$ to $A$. We now verify that the conditions of Lemma 1 are satisfied by $\epsilon_{k}$.

(1) Let $r=\sum_{i=0}^{m} r_{i} x^{i}, v=\sum_{j=0}^{n} v_{j} x^{j}$. Then $\epsilon_{k}(r v)=\sum_{i, j} \epsilon_{k}\left(r_{i} x^{i} v_{j} x^{j}\right)$ so it suffices to show (1) for the monomial cases.

Consider $\epsilon_{k}\left(a x^{i} b x^{j}\right)$ with $a, b \in A$. Inductively, $x^{i} b=\sum_{l=0}^{i} t_{l}(b) x^{l}$, where $t_{l}=$ $\sum_{w \in W_{l}} w$ and $W_{l}$ is the set of all words in $\sigma, \delta$ with $l \sqcup \sigma$ 's and $i-l \sqcup \delta$ 's. Now

$$
x^{i+1} b=\sum x t_{l}(b) x^{l}=\sum_{l} \sum_{w} x w(b) x^{l} .
$$

Since $x w(b)=(\sigma w)(b) x+(\delta w)(b), \sigma w$ is a word with $l+1 \sqcup \sigma^{\prime} s$ and $i-l \sqcup \delta$ 's and $\delta w$ is a word with $l \sqcup \sigma^{\prime} s$ and $i-l+1 \sqcup \delta$ 's; thus the general word with $l+1 \sqcup \sigma$ 's and $i-l \sqcup \delta$ 's beginning with a $\delta$ comes from a word with $l+1 \sqcup \sigma$ 's and $i-l-1 \sqcup \delta$ 's (in $W_{l+1}$ ).

Thus

$$
\epsilon_{k}\left(a x^{i} b x^{j}\right)=\epsilon_{k}\left(\sum_{l} \sum_{w} a w(b) x^{l} x^{j}\right)=\sum_{l} \sum_{w} a w(b) k^{l} k^{j} .
$$

Also, $\epsilon_{k}\left(a x^{i} \epsilon_{k}\left(b x^{j}\right)\right)=\epsilon_{k}\left(a x^{i} b k^{j}\right)=\epsilon_{k}\left(a\left(\sum_{l} \sum_{w} w(b) x^{l}\right) k^{j}\right)$. Now $x k=\sigma(k) x+\delta(k)=$ $k x$, by the definition of $K$, so $\epsilon_{k}\left(a x^{i} \epsilon_{k}\left(b x^{j}\right)\right)=\sum_{l, w} a w(b) k^{j} k^{l}$. Thus $\epsilon_{k}\left(a x^{i} \epsilon_{k}\left(b x^{j}\right)\right)=$ $\epsilon_{k}\left(a x^{i} b x^{j}\right)$ and $\epsilon_{k}\left(r \epsilon_{k}(v)\right)=\epsilon_{k}(r v)$.

(2) Suppose $\epsilon_{k}(u)=0$ for all $k \in K$. Let $u=\sum_{i=0}^{n} u_{i} x^{i}, u_{i} \in A$. Then $\sum_{i=0}^{n} u_{i} k^{i}=0$ for all $k \in K$. 
Let $k_{0}, k_{1}, \ldots, k_{n}$ be distinct elements of $K$. Then set

$$
V=\left[\begin{array}{cccc}
1 & \vdots & \vdots & 1 \\
k_{0} & \vdots & \vdots & k_{n} \\
k_{0}^{2} & \vdots & \vdots & k_{n}^{2} \\
\vdots & \vdots & \vdots & \vdots \\
k_{0}^{n} & \vdots & \vdots & k_{n}^{n}
\end{array}\right],
$$

the $(n+1) \times(n+1)$ Vandermonde matrix. Now $\left(u_{0}, \ldots, u_{n}\right) V=0$ and $V$ is invertible over the field of fractions of $K$, so $u_{0}=u_{1}=\cdots=u_{n}=0$. Hence $u=0$.

By Lemma $1_{R} R_{A}$ is reflexive.

Corollary 1 [4, Lemma 0]. If $K$ is an infinite field and $R=K[x]$, then ${ }_{R} R_{K}$ is reflexive.

The next result gives sufficient conditions for every non-torsion module to be reflexive and generalises Hadwin's result concerning non-torsion modules over $K[x]$.

THEOREM 2. Let $R$ be a K-algebra domain and suppose that $R$ has finite Goldie dimension. If ${ }_{R} R_{K}$ is reflexive, then every non-torsion $R$-module is reflexive.

Proof. Let $M$ be a non-torsion $R$-module. Let $m_{0} \in M$ be such that $r m_{0} \neq 0$ for all $0 \neq r \in R, \alpha \in$ alglat ${ }_{R} M_{K}$, and $m \in M$. Note that $R m$ is a homomorphic image of $R$ and $R m_{0} \cong R$. Thus if $R m_{0} \cap R m=0$, then $R m_{0} \oplus R m$ is reflexive as an $R, K$ bimodule by [2, Corollary 1.9]. Therefore if $\alpha m_{0}=r_{0} m_{0}$, with $r_{0}$ uniquely determined by the torsion-freeness of $m_{0}$, then $\alpha m=r_{0} m$ also.

Now suppose $R m_{0} \cap R m \neq 0$. We show first that $m$ is torsion-free. Let $0 \neq x=$ $a_{0} m_{0}=a m$ for some $a_{0}, a \in R$. If $b \in R$ and $b x=0$ then $b a_{0} m_{0}=0$, so $b a_{0}=0$ by the choice of $m_{0}$, and finally $b=0$ from the domain property. Thus $L=\{r \in R \mid r m=0\}$ is such that $R a \cap L=0$. Now $\operatorname{dim} R \geq \operatorname{dim}(R a \oplus L)=\operatorname{dim} R a+\operatorname{dim} L=\operatorname{dim} R+\operatorname{dim} L$, hence $\operatorname{dim} L=0$. Therefore $L=0$ and $m$ is torsion-free.

We now have unique $r_{0}, r \in R$ such that $\alpha m_{0}=r_{0} m_{0}$ and $\alpha m=r m$. Moreover, from the reflexivity of $R m_{0} \cong R m \cong R$ we have $\alpha x=r_{0} x=r x$ and so $r_{0}=r$ since $x$ is also torsion-free. Thus $\alpha m=r_{0} m$ and $M$ is reflexive.

Corollary 2 [4, Theorem 1]. If $K$ is an infinite field and $R=K[x]$, then every non-torsion $R$-module is reflexive.

\section{REFERENCES}

1. L. Brickman and P. A. Fillmore, The invariant subspace lattice of a linear transformation, Canad. J. Math. 19 (1967), 810-822.

2. K. R. Fuller, W. K. Nicholson and J. F. Watters, Reflexive bimodules, Canad. J. Math. 41 (1989), 592-611. 263.

3. P. R. Halmos, Reflexive lattices of subspaces, J. London Math. Soc. (2) 4 (1971), $257-$

4. D. W. Hadwin, Algebraically reflexive linear transformations, Linear and Multilinear Algebra 14 (1983), 225-233.

5. H. Leptin, Linear kompakte Moduln und Ringe, Math. Z. 62 (1955), 241-267. 